\title{
Determinación del tipo de sonrisa infantil ideal de acuerdo a la percepción de los padres y Odontopediatras
}

Judith Margarita Plata Ramirez';

Hilda H.H. Torre Martínez²;

Gustavo Israel Martínez González ${ }^{3}$

\section{Resumen}

Objetivo: Comparar la percepción de los padres de familia y Odontopediatras en relación a la sonrisa infantil ideal. Material y Métodos: 2 Grupos de evaluadores: 97 Padres de familia (grupo 1) y 99 Odontopediatras (grupo 2). Se realizaron observaciones fotográficas en 25 fotografías para determinar la percepción de ambos grupos con respecto a la sonrisa estética. Se compararon los grupos mediante la prueba chi cuadrada con $\mathrm{p}=0,05$. Resultados: Respecto a las expectativas de los padres en cuanto a estética de la sonrisa infantil prefieren una exposición moderada de nivel de teji- do gingival, diastemas y espacios interincisales; con una distancia vertical promedio. Los Odontopediatras prefieren una exposición mínima de tejido gingival, dientes juntos, sin diastemas ni espacios interincisales. Conclusiones: Se acepta la hipótesis ya que al comparar la percepción de los padres de familia y Odontopediatras en relación a la sonrisa infantil ideal existió una diferencia significativa entre la percepción visual de los padres de familia y los profesionistas con respecto a la sonrisa ideal.

Palabras clave: Percepción, sonrisa, infantil, estética, padres, odontopediatras.

Artigo Original

\section{Determinação do tipo de sorriso infantil ideal de acordo com a percepção de pais e odontopediatras.}

\section{Resumo}

Objetivo: Comparar a percepção de pais e odontopediatras em relação ao sorriso infantil ideal.
Material e Métodos: 2 Grupos de avaliadores: 97 Pais (grupo 1) e 99 Odontopediatras (grupo 2). Imagens de 25 fotografias foram entregues, aos país e odontopediatras, para determinar a per-

\footnotetext{
1. Maestría en Ciencias Odontológicas en el área de Odontopediatría, Universidad Autónoma de Nuevo León. Monterrey, México.

2. PhD en Odontología., MS en Ortodoncia., Profesora de tiempo completo., Subcoordinadora del Posgrado de Ortodoncia., Facultad de Odontología., Universidad Autónoma de Nuevo León. Monterrey, México.

3. PhD en Investigación, Profesor del Posgrado de Odontopediatría, Facultad de Odontología; Universidad Autónoma de Nuevo León. Monterrey, México.
} 
cepção em ambos os grupos quanto ao sorriso estético em crianças. Os resultados foram comparados usando o teste do qui-quadrado com $p=0,05$. Resultados: Em relação às expectativas dos pais sobre a estética do sorriso infantil, estes prefiraram uma exposição moderada do nível do tecido gengival, diastemas e espaços interincisivos; com uma distância vertical média. Odontopediatras preferiram exposição mínima do tecido gengival, dentes sem diastemas ou espaços interincisivos. Conclusões: A hipótese encontrada sobre o sorriso das crianças do ponto de vista dos país e odontopediatras é que houve uma diferença significativa entre a percepção visual em relação ao sorriso ideal.

Palavras chave: Percepção, sorriso, infância, estética, pais, odontopediatria.

Original article

\section{Determination of the ideal children's smile type according to the perception of parents and Pediatric dentists}

\section{Abstract}

Aim: To compare the perception of parents and pedodontists in relation to the ideal child smile. Material and Methods: 2 Groups of evaluators: 97 parents (group 1) and 99 pedodontists (group 2). Photographic observations were performed in 25 photographs to determine the perception of both groups regarding the aesthetic smile. The groups were compared using the chi-square test with $\mathrm{p}=0.05$. Results: Expectations of the parents regarding the aesthetics of the children's smile, they prefer moderate exposure of gingival tissue, diastemas and interincisal spacings; with an average vertical distance. Pedodontists prefer minimal exposure of gingival tissue, teeth together, without diastemas or interincisal spacings. Conclusions: The hypothesis is accepted because when comparing the perception of parents and Pedodontists in relation to the ideal child smile there was a significant difference between the visual perception of parents and professionals with respect to the ideal smile.

Key words: Perception, smile, infant, esthetics, parents, pediatric dentistry. 


\section{Introducción}

La sonrisa es legítimamente considerada un medio valioso no verbal de comunicación social y un criterio del atractivo facial; firmemente conectadas entre sí, juega un papel importante en la expresión facial y la apariencia; ${ }^{1}$ es la luz del rostro, abre muchas puertas, genera actitudes positivas y abre el camino para llegar a los demás. ${ }^{2}$

El valor de una sonrisa atractiva es indiscutible. Estudios demuestran que "las personas confían más en una persona que sonríe en comparación a una que no". ${ }^{3}$ Tener una sonrisa hermosa y juvenil es el principal objetivo que desean los pacientes. ${ }^{4}$

Muy frecuentemente los padres de familia acuden con el Odontólogo a consulta con muchas dudas y haciendo múltiples preguntas enfocadas a la estética dental de sus hijos, entre ellas involucran tamaño, espaciamiento, posición y forma.

Las respuestas que dan los Odontólogos son basadas en los parámetros teóricos; sin embargo, causa admiración que posterior al esfuerzo que se pone en devolver la salud y estética oral a sus hijos, algunos padres creen que no es lo ideal. Existen grandes cantidades de estudios en relación a la percepción estética y la sonrisa del adulto; no así en niños. En Ortodoncia la información acerca de la autopercepción de la oclusión es considerada de importancia. Relacionan auto-conciencia y satisfacción con el estatus oclusal verdadero y determinan si la insatisfacción esta basada con anomalías percibidas realísticamente. ${ }^{5}$

Las tendencias actuales demuestran gran énfasis en la estética de la sonrisa, han establecido la relación de la satisfacción con la sonrisa y las características de esta, al tomarles una fotografía con la sonrisa posada. ${ }^{6}$
Entre los estudios de autopercepción existe éste acerca del atractivo dental en los niños; creando una escala de clasificación estandarizada de atractivo dental, que pudiera ser usada para asistir la determinación de prioridades del tratamiento, para obtener una impresión realista de su atractivo dental.

Los niños que subestimaron su propio atractivo dental también tenían un bajo promedio de puntaje en su autoestima general. ${ }^{7}$ Otros estudios se basan en la percepción estética de la sonrisa mostrando imágenes predeterminadas estudiando algunos factores en la sonrisa; éstos se realizan entre personas comunes, otros son comparativos entre Odontólogos y estudiantes de Odontología, entre especialistas en alguna de las ramas de Odontología, también comparativos entre diferentes zonas geográficas para comparar las diferencias culturales. Evalúan el atractivo de una sonrisa de acuerdo a variables de normas estéticas. ${ }^{8}$ Determinan la percepción estética de hombres y mujeres a variables en la exposición superior e inferior de tejido gingival al sonreír y hablar así como a la inclinación del plano incisal. ${ }^{9}$ El manejo de espacio excesivo anterior es un problema común en la practica dental. Durante la planeación de tratamiento ortodóntico o de restauración, los clínicos deben decidir cómo distribuir este espacio. Comparan la percepción estética de 4 diferentes diastemas anteriores, incluyendo espacios primates, diastemas sugeridos por Frush y Fisher, y por Lombardi y diastema de la línea media. ${ }^{10}$

Relacionan los espacios negros entre los incisivos centrales superiores y la percepción estética de la sonrisa. ${ }^{11}$

Se encontró que las preferencias de los dentistas y los pacientes diferían significativamente al 
evaluar variables dentales de forma, simetría y proporción $^{12}$. Se ha analizado la percepción de la estética en la sonrisa y sus alteraciones en estudiantes de Odontología; para determinar si hay diferencias en la percepción entre alumnos de diferente grado, en el mismo nivel y entre géneros; así como determinar si haber recibido tratamiento ortodóntico anteriormente puede influir en su percepción. ${ }^{13}$ Se recolectaron datos respecto a la percepción de los canadienses de la sonrisa estética y se comparó con los datos de USA para poder evaluar las diferencias culturales. ${ }^{14}$ Las pocas investigaciones que se enfocan a la estética en la sonrisa de los niños son básicamente en relación a la autopercepción. Estudiaron su habilidad para auto-evaluarse y su nivel de auto-satisfacción con su propia apariencia dental. ${ }^{15}$ Encontramos también estudios respecto a la apreciación y estética de la fluorosis dental, comparando la prevalencia y severidad y evaluaron la percepción de los niños hacia sus dientes. ${ }^{16} \mathrm{Al}$ evaluar las preocupaciones acerca de la fluorosis; encontraron que los padres estaban más preocupados e insatisfechos con la apariencia dental de sus hijos que los propios niños. ${ }^{17}$ $\mathrm{Al}$ determinar la prevalencia de fluorosis dental para niños de áreas fluoradas y no fluoradas; se les dio la oportunidad a los niños y sus padres de expresar sus preocupaciones acerca de la estética dental de los niños. La calificación de los padres e hijos sugiere que hay pocos niños con problemas de percepción estética en la categoría 1 TSIF. Mientras que la preocupación de los padres era más común. Sin ser una sorpresa, los niños con fluorosis con valores entre 2-6 TSIF parecen tener mayores preocupaciones sobre el color dental. ${ }^{18}$ Por último encontramos algunos otros estudios pero relacionados a la percepción de la caries dental; con respecto al atractivo, percepción de salud y preferencias de tratamiento de los incisivos primarios superiores, ${ }^{19}$ y otros relacionados con los materiales de rehabilitación, en este estudio evaluaron las preferencias estéticas en relación con el color y la forma de coronas utilizadas para dientes incisivos superiores primarios. ${ }^{20}$

Una de las características de la estética facial que sin duda resalta a la vista es la sonrisa. Las mejorías estéticas son requeridas frecuentemente en los consultorios dentales. ${ }^{2}$ Los padres están ahora involucrados en la toma de decisiones, las cuales previamente eran responsabilidad de los profesionistas médicos o dentistas. La toma de decisiones clínicas se ha convertido en un proceso social que incluye dentista, paciente y en ocasiones otros miembros de la familia. Un plan de tratamiento raramente ofrece un sólo tipo de tratamiento sin alternativas. Los dentistas deben considerar la postura de los padres, creencias y valores con respecto a la estética y su función cuando presentan opciones de tratamiento. Establecer cual es el resultado de mayor importancia para todos los involucrados así como cuales son los resultados más probables de cada uno de estos es para proveer salud bucal de calidad, ya que la opinión de los dentistas y los padres del tratamiento puede variar, entender estas diferencias puede mejorar las comunicaciones y ayudar en la elección de los planes de tratamiento. ${ }^{19}$

Hipótesis: Existe una diferencia entre la percepción visual de los padres de familia y los profesionistas con respecto a la sonrisa ideal.

El Objetivo general fue comparar la percepción de los padres de familia y Odontopediatras en relación a la sonrisa infantil ideal. Objetivos Específicos: Conocer cuáles son las expectativas de los padres en cuanto a estética de la sonrisa infantil; determinar el grado de acuerdo o desacuerdo de los padres y Odontopediatras. 


\section{Materiales y Métodos}

2 Grupos de evaluadores: 97 Padres de familia (grupo 1) y 99 Odontopediatras (grupo 2). Se encuestó a los padres de familia que acudieron a consulta del Posgrado de Odontopediatría de la Universidad Autonóma de Nuevo León (UANL) los cuales no tenían relación con el ambiente odontológico. Los Odontopediatras catedráticos, egresados del Posgrado de Odontopediatría de la (UANL) y miembros de la Academia Mexicana de Odontopediatría (AMOP). Siguiendo y respetando el código ético de Helsinki la norma 013 del Código Sanitario Mexicano y de la Secretaria de Salud, se describen las 5 variables a estudiar y en cada uno se muestran 5 fotografías de sonrisas, en las cuales la sonrisa original es tomada como control o de $0 \mathrm{~mm}$ y se van aumentando de 0,5 mm hasta llegar a 2,00 mm cada una de las 5 variables, las fotografías fueron puestas al azar sin orden ni secuencia lógica para que no se predeterminaran las respuestas (Figuras 1 a 5). Las encuestas, se utilizaron como hoja de recolección de datos y todos los datos se vaciaron en

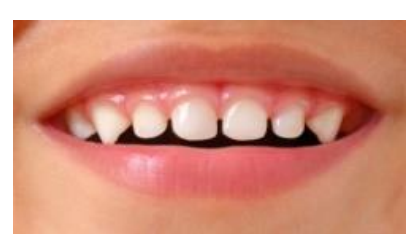

1) $2,0 \mathrm{~mm}$.

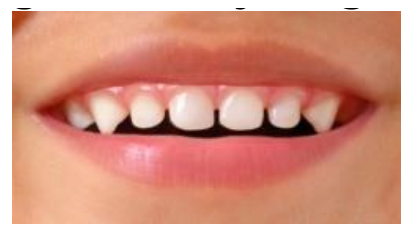

3) $0,5 \mathrm{~mm}$.

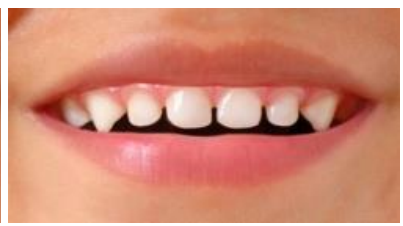

2) Control

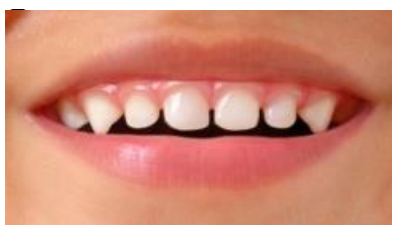

4) $1,0 \mathrm{~mm}$.

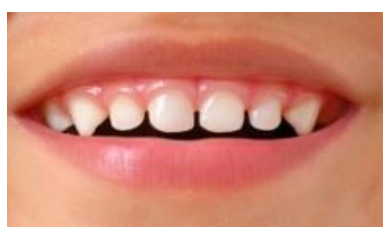

5) $1,5 \mathrm{~mm}$.

Figura 1. Tejido gingival: Encía expuesta arriba de los dientes.

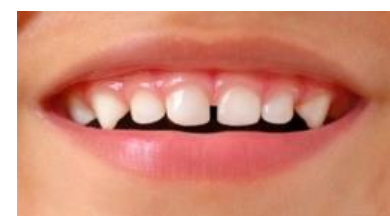

1) $1,5 \mathrm{~mm}$.

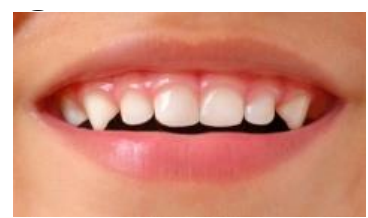

3) Control.

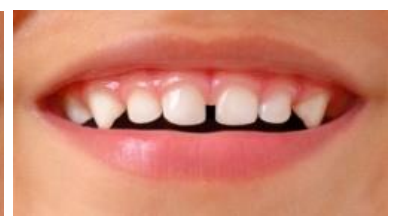

2) $2,0 \mathrm{~mm}$.

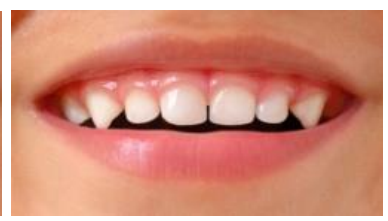

4) $0,5 \mathrm{~mm}$.

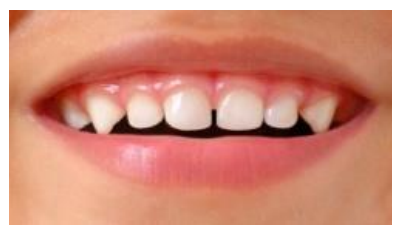

5) $1 \mathrm{~mm}$.

Figura 2. Diastema: Espacio encontrado entre los 2 dientes centrales.

una hoja que se diseñó para este fin de Microsoft Excel de manera simple. Posteriormente se realizó el análisis estadístico con el programa SPSS versión 22 y se utilizó la prueba estadística Chi cuadrada con 95\% de confiabilidad; y el valor de $\mathrm{p}=0,05$.

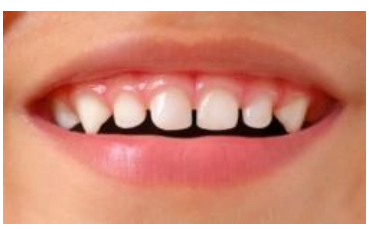

1) $1,0 \mathrm{~mm}$.

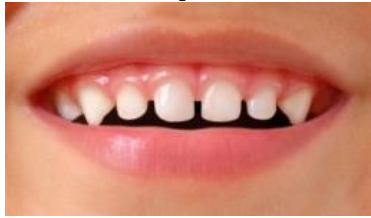

3) $1,5 \mathrm{~mm}$.

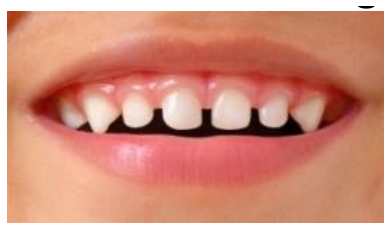

2) $2,0 \mathrm{~mm}$.

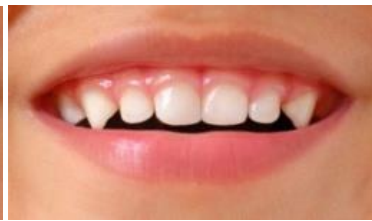

4) Control

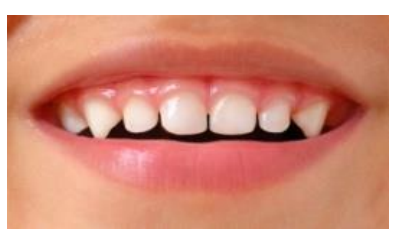

5) $0,5 \mathrm{~mm}$.

Figura 3. Espacios interdentales: Espacios encontrados entre los dientes. 


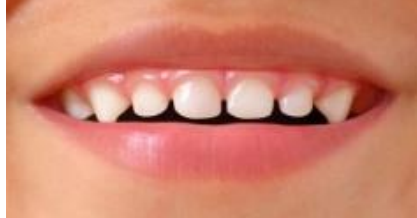

1) $0,5 \mathrm{~mm}$.

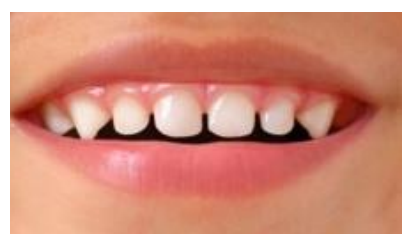

3) $1,5 \mathrm{~mm}$.

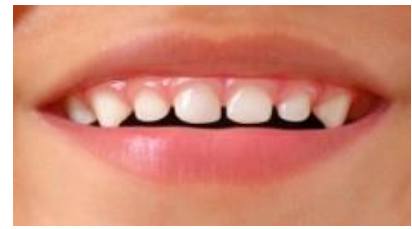

5) Control

Figura 4. Distancia vertical: Distancia de los dientes de arriba abajo.

\section{Resultados}

Población 196 participantes de los cuales fueron 153 (78\%) de género femenino y 43 (22\%) de género masculino, observándose un predominio de Odontopediatras femeninas de $87.88 \%$, al igual que de madres en un $68.04 \%$, con una relación de 8 de cada 10 de ambos grupos son femeninos (Gráfico1).

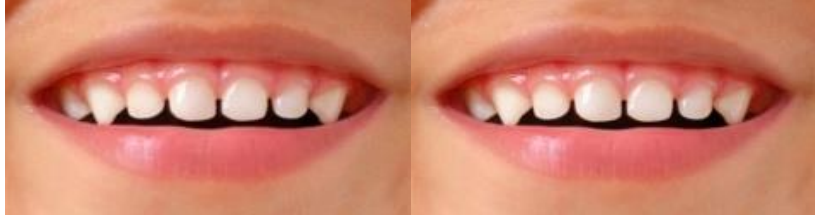

1) Control

2) $0,5 \mathrm{~mm}$.

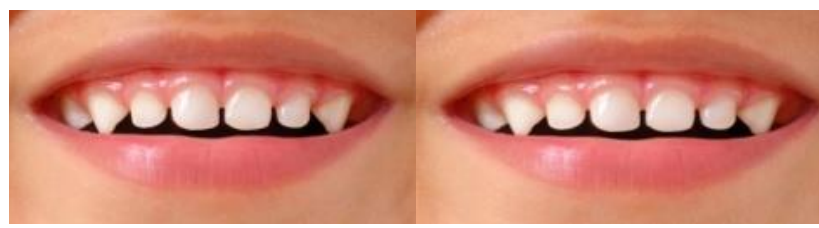

3) $1 \mathrm{~mm}$.

4) $1,5 \mathrm{~mm}$.

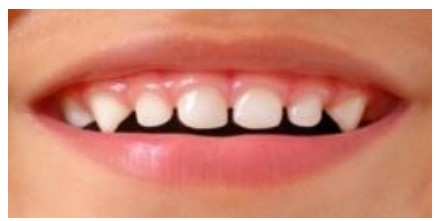

5) $2,0 \mathrm{~mm}$.

Figura 5. Espacios primates: Espacio encontrado entre los colmillos y su diente vecino más cercano al centro.

La edad mínima fue de 19 años y máxima 71 años edad promedio de 35 .

Tejido Gingival se observa una diferencia significativa de apreciación técnicamente, ya que ambos grupos eligieron en su mayoría la imagen \#2 y la \#3 las cuales varían sólo 0,5 mm; realmente no hubo discrepancias significativas entre ambos grupos al evaluar la distancia de encía a la-

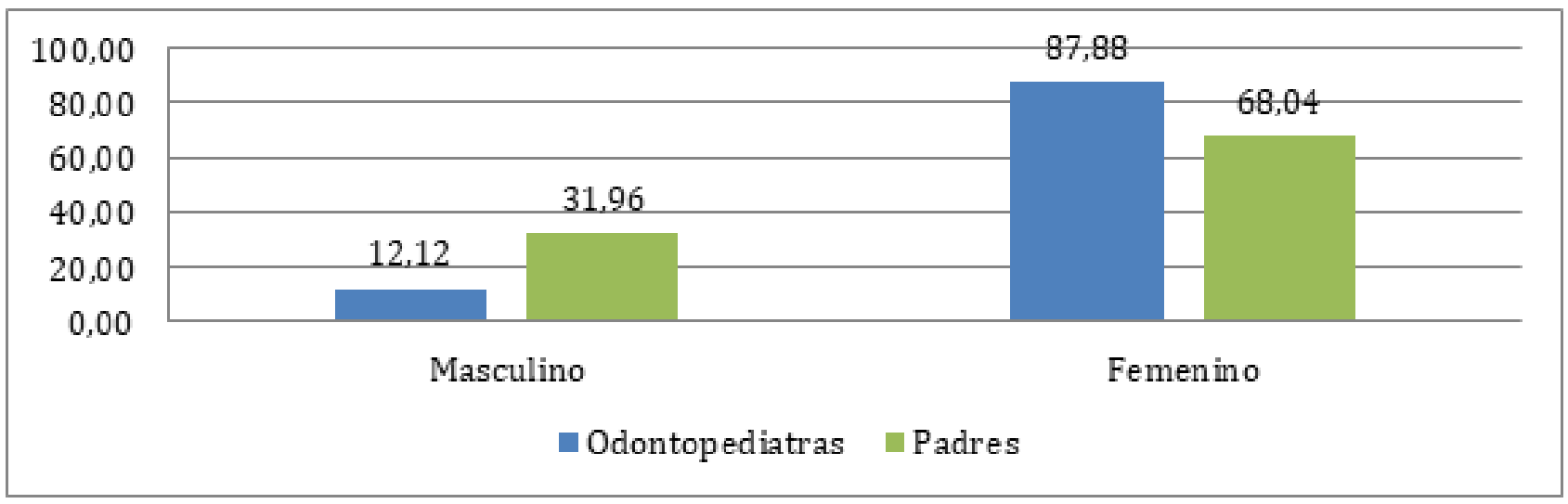

Gráfico 1. Distribución de las muestras por género. 


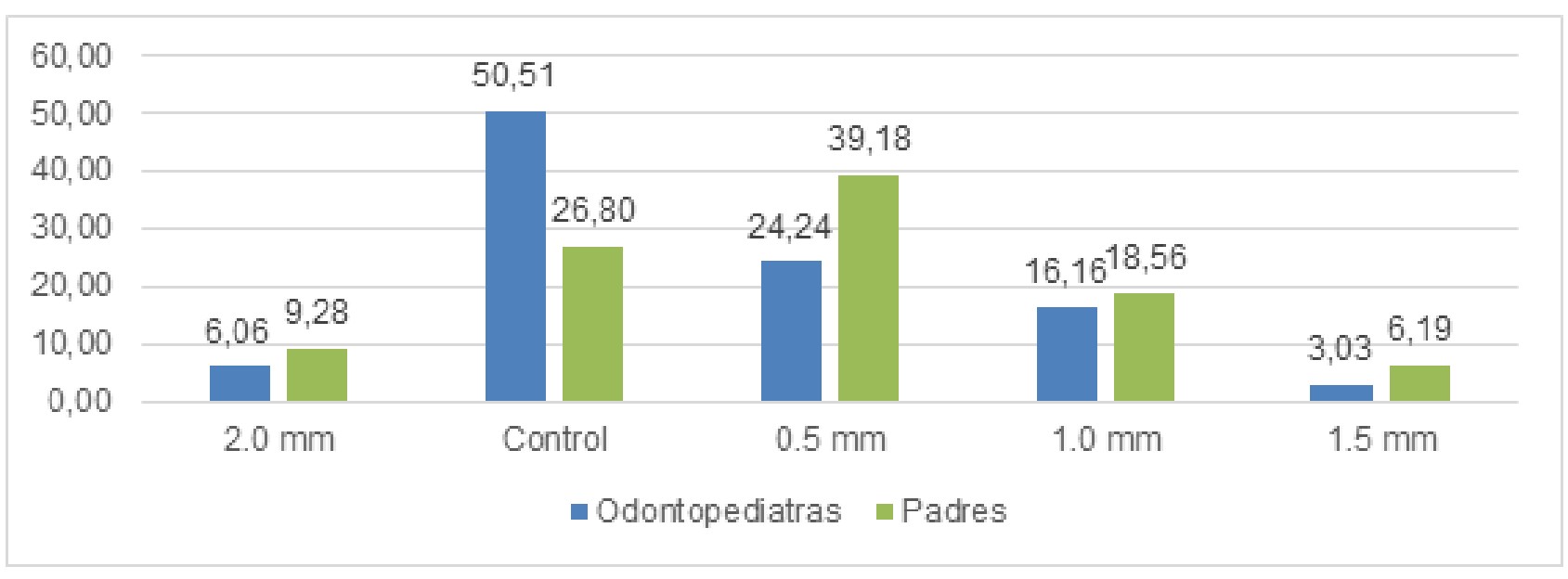

$X^{2}=12.43, p=0.0143$

Gráfico 2. Percepción del nivel del tejido gingival, comparativo entre padres y odontopediatras.

bio. Los Odontopediatras calificaron como más atractivo el nivel de tejido gingival en la imagen \#2 (el control) que mostraba un nivel mínimo de tejido gingival, con un 50\% de acuerdo (50 de 99 menciones). Los padres discernieron más eligiendo la imagen \#3 (+0,5 mm) con un 39\% (38 de 97 menciones). Hubo diferencias estadísticamente significativa $(p=0,0143)$ (Gráfica 2).

Diastema Mientras que los Odontopediatras eligieron la fotografía \#3 (una vez mas el con- trol) el cual era de 0mm con 63\% (63 de 99 menciones) para los padres la \#5 de $+1,00 \mathrm{~mm}$ de separación con $41 \%$ (40 de 97 menciones). Hubo diferencias estadísticamente significativa ( $p=0,0001)$. (Gráfica 3).

Espacios Interdentales los Odontopediatras coincidieron significativamente en un 66\% (66 de 99 menciones) eligiendo la imagen \#4 (el control) que no muestra espacios interdentales. Los padres de familia eligieron la imagen $\# 1$ con espa-

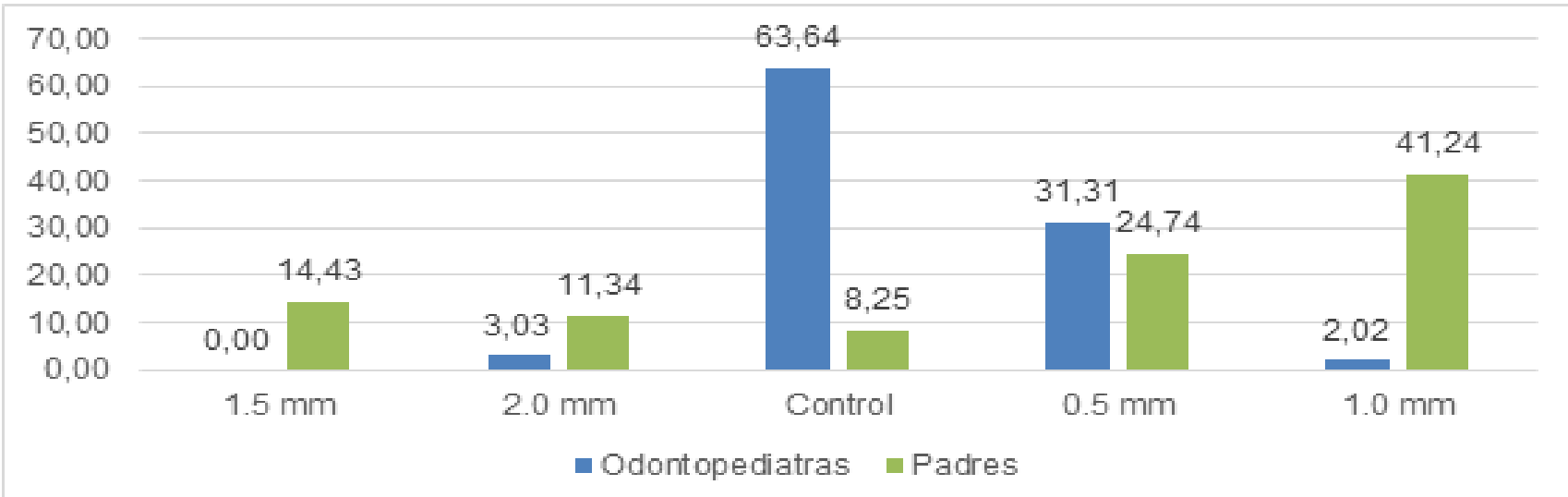

$x^{2}=96,43, p=0,0001$

Gráfico 3. Percepción del diastema, comparativo entre padres y odontopediatras. 


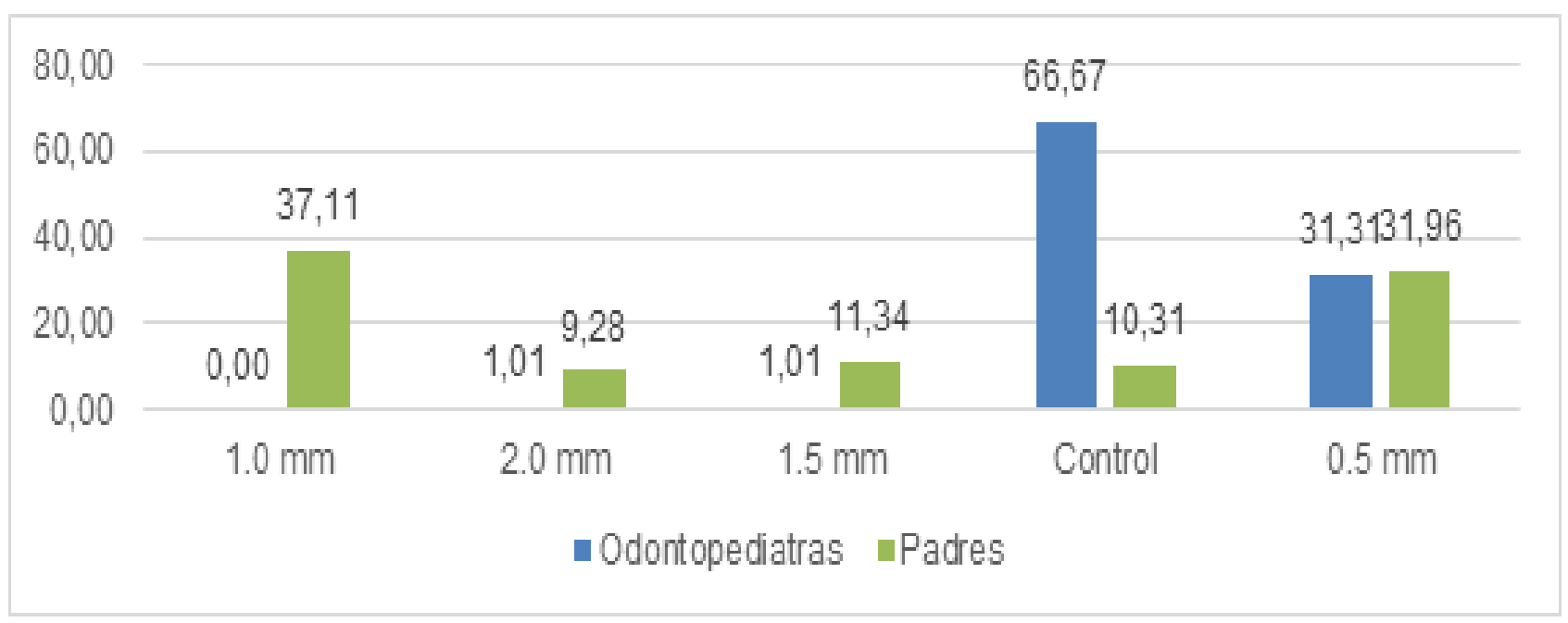

$X^{2}=91,98, p=0,0001$

Gráfico 4. Percepción de los espacios interdentales, comparativo entre padres y odontopediatras.

cios interdentales de 1,00mm 37\% (36 de 97 menciones); la $2^{\mathrm{a}}$ elección para ambos grupos fue la imagen \#5 con espacios de $0,5 \mathrm{~mm}$ difiriendo de la imagen ganadora con sólo $0,5 \mathrm{~mm}$.

Hubo diferencias estadísticamente significativa $(\mathrm{p}=0,0001)$. (Gráfico 4).
Distancia Vertical los Odontopediatras eligieron como más estética 2 imágenes con igual número de menciones la \#3 de $+1.5 \mathrm{~mm}$ con $24 \%$ y la \#4 de $+2,00 \mathrm{~mm}$ Los padres eligieron la imagen $\# 2+1,00 \mathrm{~mm}$ con $35 \%$; ambos grupos mostraron discrepancias significativas en este punto. $(\mathrm{p}=0,0637)$. (Gráfico 5).

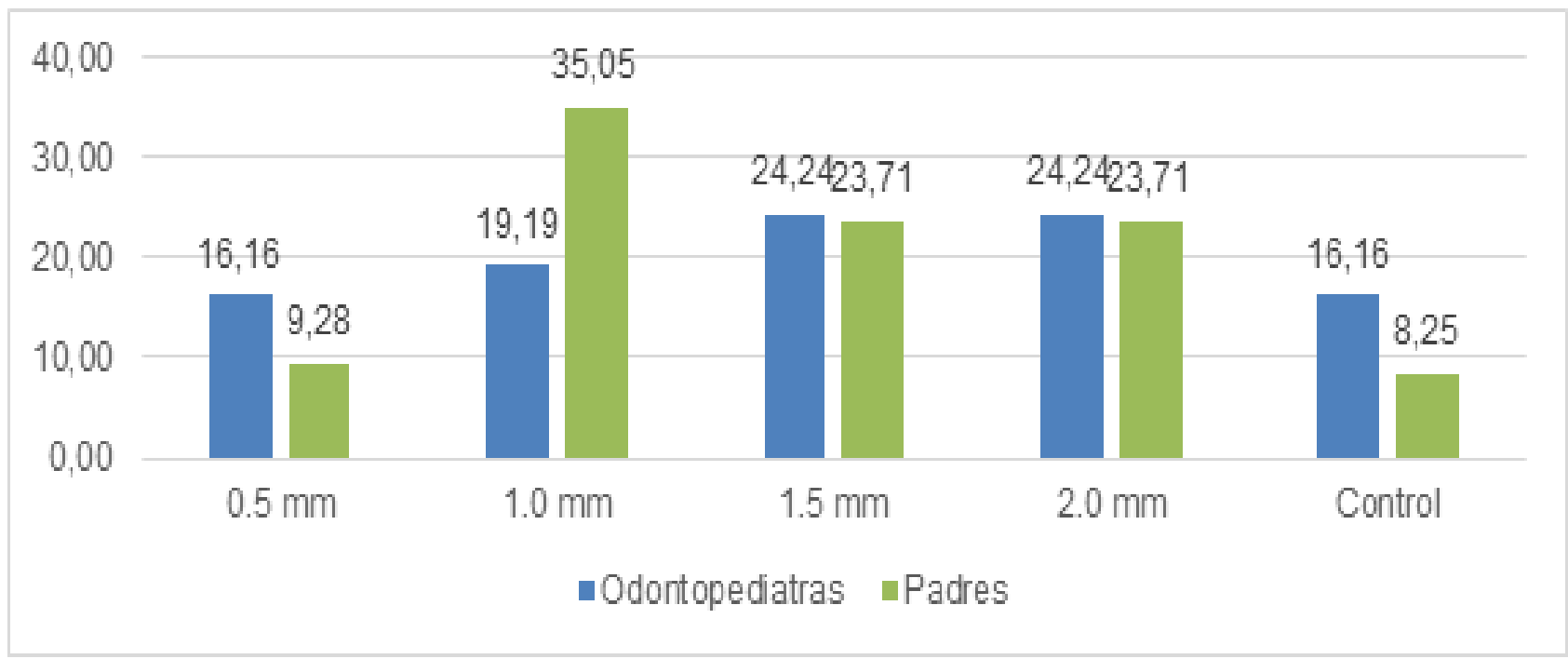

$X^{2}=8,89, p=0,0637$

Gráfico 5. Percepción de la distancia vertical o coronal clínica, comparativo entre padres y odontopediatras. 


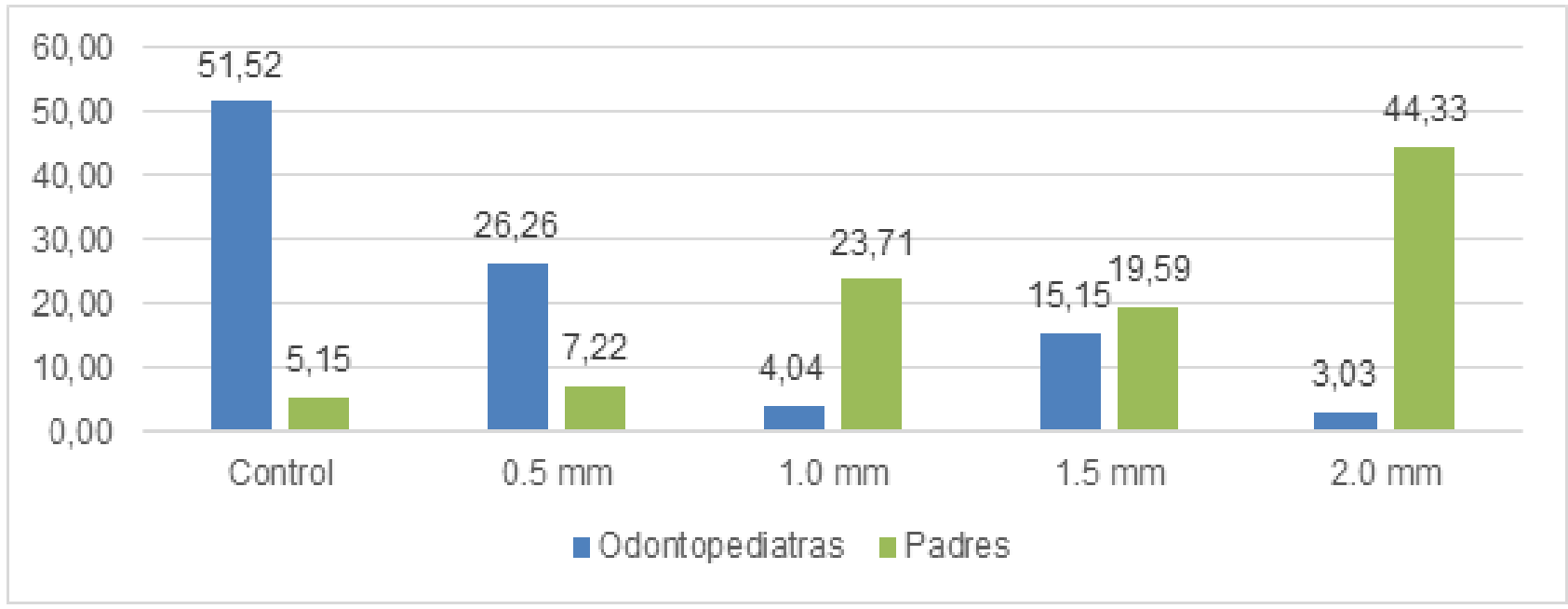

$X^{2}=97.33, p=0.0001$

Gráfico 6. Percepción de los espacios primates, comparativo entre padres y odontopediatras.

Espacios Primates hubo diferencias significativas entre ambos grupos ( $\mathrm{p}=0,0001)$ eligiendo los 2 opuestos. Los Odontopediatras eligieron la imagen \#1 0mm (Control) 51\% (51 de 99 menciones) coincidiendo significativamente entre ellos y los padres la \#5+2,00 mm 44\% (43 de 97 menciones). (Gráfico 6, Figuras 6 a 8)

\section{Discusión}

El propósito de la presente investigación fue determinar el tipo de sonrisa infantil ideal de acuerdo a la percepción de los padres y Odon-

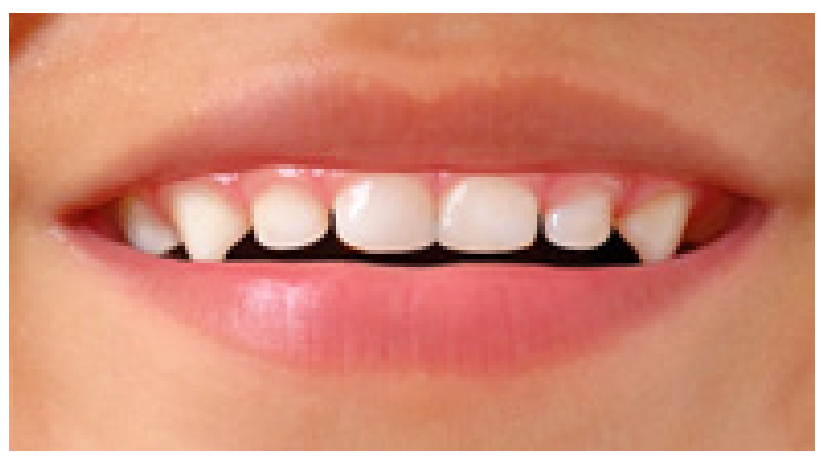

Figura 6. Imagen Ideal (De acuerdo a moda). topediatras, así como comparar la percepción de los padres de familia y Odontopediatras en relación a la sonrisa infantil ideal. La hipótesis fue que existe una diferencia entre la percepción visual de los padres de familia y los profesionistas con respecto a la sonrisa ideal.

\section{Percepción del nivel del tejido gingival}

Los Odontopediatras calificaron como más atractivo el nivel de tejido gingival en la imagen \#2 (la cual era el control) que mostraba un nivel mínimo de tejido gingival. Nuestros resul-

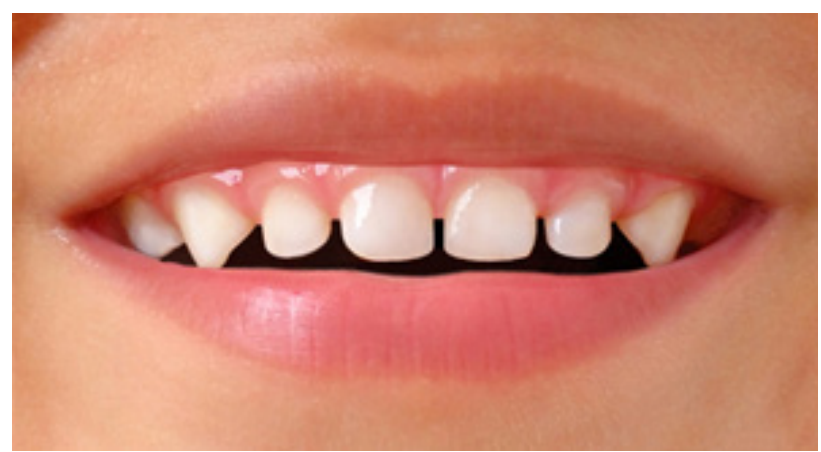

Figura 7. Imagen ideal padres. 
tados coinciden con Gerón, ${ }^{9}$ García Ruiz, ${ }^{2}$ Kokich, ${ }^{21}$ Hunt, ${ }^{22}$ Kokich, ${ }^{23}$ cuyos resultados fueron que mostrar $1 \mathrm{~mm}$ o más de encía marginal, ya era antiestético. Los padres discernieron más eligiendo la imagen \#3 (+0,5 $\mathrm{mm})$ en la cual se aprecia moderadamente la encía.

\section{Percepción del diastema}

Mientras que los Odontopediatras eligieron una vez mas el control $(0 \mathrm{~mm})$, los padres la \#5 de $+1,00 \mathrm{~mm}$ de separación. Nuestros resultados difieren con Kokich ${ }^{21}$, Kokich ${ }^{23}$ sus resultados fueron que dentistas y personas comunes no les desagrada el diastema de la línea media hasta que la distancia de los puntos de contacto de los incisivos centrales es mayor a 2,00mm y en los Ortodoncistas de $1.5 \mathrm{~mm}$ Es sorprendente como los padres si coinciden con estos y no así los Odontopediatras.

Los Odontopediatras coinciden con Noureddine ${ }^{10}$ quien encontró que las personas comunes calificaron como más atractiva la sonrisa que presentaba espacios primates y la menos atractiva el diastema de la línea media. Recalcando así que las personas comunes se inclinan hacia el menor espacio visible. Por el otro lado los padres de nuestra investigación difieren con éste.

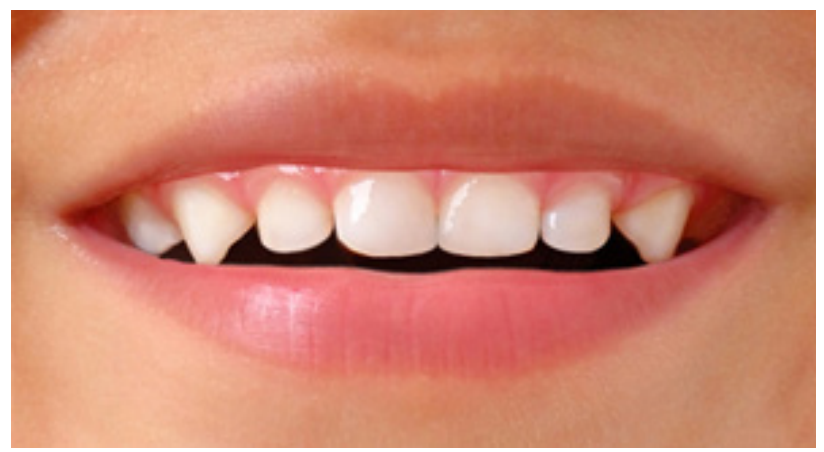

Figura 8. Imagen ideal odontopediatras.

\section{Percepción de espacios interdentales}

Los Odontopediatras eligen la imagen que no muestra espacios interdentales. Los padres de familia eligieron la imagen con espacios interdentales de 1,00mm; cabe mencionar que la $2^{2}$ elección para ambos grupos fue la imagen con espacios de 0,5 mm difiriendo de la imagen ganadora con sólo $0,5 \mathrm{~mm}$.

Nuestros resultados difieren relativamente con Woo,19 en este, los dentistas calificaron como más estéticos los incisivos con ligera separación (52\%) y los padres las que no presentaban espacios (69\%); Los dentistas calificaron en $2^{\circ}$ lugar los que no presentaban espacios (49\%) y los padres a los espacios ligeros como $2^{\circ}$. En los nuestros se presenta esta misma discrepancia en la cual se eligieron en ambos grupos un $1^{\circ}$ y $2^{\circ}$ lugar marcados en los que las variables fueron de igual manera nula y mínima separación interdental pero en estos eligieron inversamente a el anterior estudio los Odontopediatras prefirieron sin espacios interdentales mayormente y los padres con ligeros espacios.

\section{Percepción distancia vertical}

Los Odontopediatras eligieron 2 imágenes la \#3 de $+1.5 \mathrm{~mm}$ con $24 \%$ y la \# $4 \mathrm{de}+2,00 \mathrm{~mm}$ con $24 \%$. Los padres eligieron la imagen $\# 2+1,00 \mathrm{~mm}$. Cabe mencionar que ésta es un punto medio entre ambas elecciones de Odontopediatras. Nuestros resultados de los Odontopediatras coinciden con Dunn, ${ }^{24}$ pero en este estudio con personas comunes y la percepción de la sonrisa, la mayoría de los grupos 24 de 25 eligieron como más atractiva la sonrisa de una mujer la cual mostraba una gran exposición de dientes; favorecieron las sonrisas que mostraban una exposición moderada dental; y en nuestra investigación 
los padres (personas comunes) se inclinan por una exposición dental menor.

\section{Percepción espacios primates}

Hubo diferencias significativas entre ambos grupos ( $p=0,0001)$., eligiendo los 2 opuestos, Odontopediatras el menor espacio y padres el mayor espacio. Los Odontopediatras eligieron la imagen \#1 ( $0 \mathrm{~mm}$ Control) coincidiendo significativamente entre ellos y los padres la $\# 5+2,00 \mathrm{~mm}$. Las respuestas del grupo de padres que formaron parte de nuestra investigación coinciden con los resultados de Noureddine, ${ }^{10}$ estudiaron la percepción de personas comunes hacia 4 diferentes tipos de diastemas modificados en computadora de una imagen de sonrisa, en este calificaron como más atractiva la sonrisa que presentaba espacios primates y la menos atractiva el diastema de la línea media. Recalcando así que las personas comunes se inclinan hacia el menor espacio visible. Los resultados de la población de estudio mostraron que existe diferencia significativa en la comparación de la percepción estética de la sonrisa infantil ideal entre padres y Odontopediatras, habiendo sido 25 imágenes mostradas. Este estudio representa una prueba de la hipótesis. Los Odontopediatras fueron más críticos que los padres de familia al evaluar las discrepancias. Los padres de familia fueron menos discriminativos de las alteraciones mínimas. Es importante mencionar que aun cuando se realizó una profunda búsqueda en las bases de datos, no encontramos estudios parecidos al nuestro. Las publicaciones revisadas difieren con los nuestros ya que son en su mayoría en adultos $\mathrm{y}$ los pocos que encontramos en niños difieren de las características de este.

\section{Conclusiones}

Se acepta la hipótesis ya que al comparar la percepción de los padres de familia y Odontopediatras en relación a la sonrisa infantil ideal existió una diferencia significativa entre la percepción visual de los padres de familia y los profesionistas con respecto a la sonrisa ideal. Respecto a las expectativas de los padres en cuanto a estética de la sonrisa infantil prefieren una exposición moderada de nivel de tejido gingival, diastemas y espacios interincisales; con una distancia vertical promedio. Los Odontopediatras prefieren una exposición mínima de tejido gingival, dientes juntos sin diastemas ni espacios interincisales.

\section{Referencias bibliográficas}

1. Maulik C, Nanda R. Dynamic smile analysis in young adults. Am J Orthod Dentofacial Othop. 2007;132: 307-15.

2. García Ruiz EP. Percepción estética de la sonrisa con respecto a la línea media y sonrisa gingival entre cirujanos dentistas, estudiantes de estomatología y personas comunes. UPAORep 2017.

3. Fernandez S. Análisis de la sonrisa y patrón facial en estudiantes de la Universidad Nacional Mayor de San Marcos. UNMSM. 2008.

4. Hulsey M. An esthetics evaluation of lip-teeth relationships present in the smile. Am. J Orthod 2000; 57: 132-44.

5. Espeland LV, Stenvik A. Perception of personal dental appearance in young adults: Relationship between occlusion, awareness, and satisfaction. Am J Orthod Dentofacial Orthop 1991; 100: 234-41.

6. Koenig Maunsell R, Lavado Torres A, Aguado Donayre J, Altamirano Quicaño M, Gallardo Barrera G, Ramos Neglia E. 
Características de la sonrisa y nivel de satisfacción en estudiantes de la Facultad. Revista Kiru. 2009; 6: 88-102.

7. Evans R, Shaw W. Preliminary evaluation of an illustrated scale for rating dental attractiveness. Eur J Orthod. 1987; 9: 314-8.

8. Tupinambá Rodrigues CD, Magnani R, Candido Machado MS, Batista Oliveira OJr. The Perception of Smile Attractiveness. Angle Orthod 2009; 79: 634-9.

9. Geron S and Atalia W. Influence of Sex on the Perception of Oral and Smile Esthetics with Different Gingival Display and Incisal Plane Inclination. Angle Orthod. 2005; 75: 778-84.

10. Noureddine A, Fron Chabouis H, Parenton S, Lasserre JF. Laypersons' esthetic perception of various computer-generated diastemas: A pilot study. J Prosthet Dent. 2014; 112: 914-20.

11. Pithon MM, Bastos GW, Miranda NS, Sampaio T, Ribeiro TP, Gomes do Nascimento LEA, da Silva Coqueiro RS. Esthetic perception of black spaces between maxillary central incisors by different age groups. Am J Orthod Dentofacial Ortop. 2013; 143: 371-5.

12. Brisman AS. Esthetics: A Comparison of Dentists' and Patients' Concepts. JADA 1980; 100: 345-52.

13. España P, Tarazona B, Paredes V. Smile esthetics from odontology students' perspectives. Angle Orthod. 2014; 84: 214-24.

14. McLeod C, Fields HW, Hechter F, Wiltshire W, Wellington RJr. and Christensen J. Esthetics and smile characteristics evaluated by laypersons. Angle Orthod. 2011; 81: 198-205.

15. Graber LW, Lucker GW. Dental esthetic self-evaluation and satisfaction. Am J Orthod Dentofacial Orthop. 1980; 77: $163-73$.

16. Dos Santos AP, Malta MC, de Marsillac MW, de Oliveira BH. Fluoride Varnish Applications in Preschoolers and Dental Fluorosis in Permanent Incisors: Results of a Nested-cohort Study Within a Clinical Trial. Pediatr Dent. 2016; 38: 414-18.

17. Furtado GE, Sousa ML, Barbosa TS, Wada RS, Martínez-Mier EA, Almeida ME. Perceptions of dental fluorosis and evaluation of agreement between parents and children: validation of a questionnaire. Cad Saude Publica. 2012; 28: 1493-505.

18. Clark DC, Hann HJ, Williamson MF, Berkowitz J. Aesthetic concerns of children and parents in relation to different classifications of the Tooth Surface Index of Fluorosis. Community Dent Oral Epidemiol. 1993; 21: 360-4.

19. Woo D, Sheller B, Williams B, Mancl L, Grembowski D. Dentists' and Parents' Perceptions of Health, Esthetics, and Treatment of Maxillary Primary Incisors. Pediatr Dent. 2005; 27: 19-23.

20. Ramírez Peña HA, Rangel Padilla EE, Martínez-Menchaca HR, Rivera Silva G, Arredondo Campo G, Barba Borrego N, Gustavo Israel Martínez GI, Valencia Hitte R. Evaluación estética de seis tipos de coronas para dientes primarios. Rev Latinoam Odontoped. 2017; 7: 6-15.

21. Kokich VO Jr, Kiyak HA, Shapiro PA. Comparing the perception of dentists and lay people to altered dental esthetics. J Esthet Dent. 1999;11: 311-24.

22. Hunt $\mathrm{O}$, Johnston $\mathrm{C}$, Hepper $\mathrm{P}$, Burden D, Stevenson M. The influence of maxillary gingival exposure on dental attractiveness ratings. Eur J Orthod. 2002; 24: 199-204.

23. Kokich VO, Kokich VG., Asuman KH. Perceptions of dental professionals and laypersons to altered dental esthetics: Asymmetric and symmetric situations. Am J Orthod Dentofacial Orthop. 2006; 130: 141-51.

24. Dunn WJ, Murchison DF, Broome JC. Esthetics: patients' perceptions of dental attractiveness. J Prosthodont. 1996; 5: 166-71.

Recibido: 26/11/2017

Aceptado: 20/02/2018

Correspondencia: Judith Margarita Plata Ramírez. kidsdentalcaremx@gmail.com. 